\title{
Polymeric nanosystems for near-infrared multispectral photoacoustic imaging: Synthesis, characterization and in vivo evaluation
}

\author{
Kegang Liu ${ }^{\mathrm{a}, 1}$, Xueya Wang ${ }^{\mathrm{a}, 1}$, Vasilis Ntziachristos ${ }^{\mathrm{c}, \mathrm{d}}$, Stephan Marsch ${ }^{\mathrm{a}}$, Patrick Hunziker ${ }^{\mathrm{a}, \mathrm{b}, *}$ \\ ${ }^{a}$ Medical Intensive Care Unit, University Hospital Basel, Petersgraben 4, 4031 Basel, Switzerland \\ ${ }^{\mathrm{b}}$ CLINAM Foundation for Nanomedicine, Alemannengasse 12, Basel, Switzerland \\ ${ }^{\mathrm{c}}$ Helmholtz Zentrum München, Institute for Biological and Medical Imaging, Ingolstaedter Landstrasse 1, Neuherberg, D-85746, Germany \\ ${ }^{\mathrm{d}}$ Technische Universität München, Chair for Biological Imaging, Ismaninger Street 22, Munich 81675, Germany
}

\section{A R T I C L E I N F O}

\section{Article history:}

Received 29 January 2016

Received in revised form 8 March 2016

Accepted 8 March 2016

Available online $\mathrm{xxxx}$

\section{Keywords:}

Amphiphilic copolymer

Nanoparticle

Photosensitizer

Photoacoustic agents

Biodistribution

Theranostic

\begin{abstract}
A B S T R A C T
Photoacoustic imaging (PAI) is a new biomedical imaging modality based on lighttriggered ultrasound emission. For in vivo application, materials with good photoacustic response to illumination in the near-infrared spectrum and suited tissue delivery strategies are needed. We developed polymeric, near-infrared responsive nanomaterials tuned for in vivo application based on oxazoline block copolymer chemistry by living cationic polymerization and a related functional transformation, loaded with a new photonic material, hydrophobized phthalocyanine Zinc complex (H-PcZn), that was efficiently encapsulated into the nanoparticles by self-assembly. The resulting nanoparticles P-NPs and N-NPs bear positive, and negative surface charge, respectively. After physicochemical characterization, applicability of the two nanoparticles as photoacoustic contrast agents was evaluated in vitro and in phantom experiments, where they exhibited excellent PAI contrast. In vivo distribution and visualization of P-NPs and N-NPs following i.v. injection imaged by PAI was confirmed by cryosection fluorescence analysis and showed that the materials accumulated in tissues within $1 \mathrm{~h}$ with differential tissue distribution. This pilot study thus describes synthesis of a novel polymeric photoacoustic nanosystem and demonstrates its potential for multimodal, photoacoustic in vivo imaging and for fluorescence imaging.
\end{abstract}

(c) 2016 Elsevier Ltd. All rights reserved.

\section{Introduction}

Photoacoustic imaging/Optoacoustic (PAI/OA) is a powerful and noninvasive imaging modality which can visualize organs and diseased sites at high penetration depths with improved spatial resolution and excellent image contrast [1-3]. PAI is therefore rapidly evolving towards future clinical applications [4-7]. For PAI, endogenous or exogenous contrast agents are exposed in the biological tissue to short near infrared (NIR) light impulses and absorb them [8,9]. Absorption leads to very localized heat production followed by thermoelastic expansion of the tissue to provide corresponding acoustic signals, which can be detected by ultrasound transducers. The resulting signals can increase penetration depth $(5-6 \mathrm{~cm})[5,6,10-12]$ compared to fluorescence and have a better resolution compared to optical imaging $(20-200 \mu \mathrm{m}$ vs. $1-2 \mathrm{~mm})[1,13]$. Availability of suited contrast agents plays a major role for the successful evolution of PAI. For example, endogenous

\footnotetext{
* Corresponding author at: Medical Intensive Care Unit, Petersgraben 4, 4031 Basel, Switzerland.

E-mail address: Patrick.Hunziker@usb.ch (P. Hunziker).

1 These authors contributed equally to the work.
} characterization and in vivo evaluation, Eur. Polym. J. (2016), http://dx.doi.org/10.1016/j.eurpolymj.2016.03.008 
chromophores such as hemoglobin and melanin have been used as contrast agents for PAI in certain applications [14-16]. However, due to the relatively low intensity of the PA signal and the specific requirements of disease detection, in particular in tumor imaging, contrast agents with optimized photoacoustic properties and suited biodistribution are required for good results in imaging. A number of such contrast agents have been designed and applied to the study of PAI in recent years, including as metal nanoparticles (gold) [17-20], $\pi$-conjugated carbon materials (SWNTs) [21-23] and their hybrids [24] as well as NIR dyes [13,25-28]. For future clinical application of such agents, availability, toxicity and biodistribution are key considerations beyond physical efficacy.

Polymeric nano-systems are attractive due to their flexibility in design, synthesis, modification and functionalization $[29,30]$. Most importantly, biocompatible and/or biodegradable versions of such systems can be designed [31]. Passive or active accumulation of nanoparticles by the enhanced permeability and retention (EPR) effect or receptor targeted uptake via the interaction between ligands and receptors to certain diseased sites such as tumor, could significantly increase the amount of payload at the targeted site [32]. We hypothesized that a polymeric nanosystem loaded with NIR detecting probes could represent an ideal contrast agent for PAI. Nanomaterials from amphiphilic copolymer have a high cargo capacity and can hide the hydrophobic NIR functional groups in the hydrophobic core of the self-assemblied nanoparticles, thereby minimizing undesired physical interactions of the photonic material with the organism, while the nanoparticle shell can be functionalized for tissue distribution control [33].

We have previously reported a promising biocompatible triblock copolymer nanosystem, poly(2-methyl-2-oxazoline)block-poly(dimethylsiloxane)-block-poly(2-methyl-2-oxazoline) (PMOXA-b-PDMS- $b$-PMOXA) for delivering hydrophobized photosensitizers for photodynamic therapy (PDT) [34]. Fluorescent payloads are frequently reported for fluorescence imaging [35] and PDT [36], but are not yet widely used as PAI contrast agents [8,9,37-39]. Conventional fluorescence imaging is limited by low fluorescence quantum yields, photobleaching and autofluorescence, problems for which PAI may have advantages. Recently, Olivo et al. demonstrated that various classes of photosensitizers can be used as efficient novel contrast agents for PAI [8,9]. Among those photosensitizers, phthalocyanines, the second-generation of porphyrin-based photosensitizers, and their metal complexes proved to be efficient due to excellent relative PA quantum yields. Here, we therefore examined the value of PMOXA- $b$-PDMS- $b$-PMOXA/H-PcZn based nanoparticles as PAI contrast agents for in vivo imaging. To the best of our knowledge, this is the first time where an amphiphilic polymeric nanoparticle with the phthalocyanine zinc complex is used for PAI. We first describe the preparation of copolymer PMOXA- $b$-PDMS- $b$-PMOXA and hydrophobized phthalocyanine zinc complex as well as the resulting nanoparticles. Next, we evaluated the PAI performance of the nanomaterial in a scattering phantom. Last, we further tested in vivo PA activity by injecting the resulting nanoparticles intravenously into mice and subsequently monitoring the biodistribution over time. On the basis of the high efficacy in PDT and PAI, the copolymer/photosensitizer-based nanoparticles as PAI contrast agents offer great potential for in vivo imaging and may be suited for disease diagnosis, e.g. in cancer, and for therapy.

\section{Materials and methods}

\subsection{Materials and instrumentations}

All chemical substances were purchased from Sigma-Aldrich (St. Gallen, Switzerland) and abcr (Karlsruhe, Germany) and were used as received unless otherwise stated. 2-Methyl-2-oxazoline, chloroform, and acetonitrile were dried by refluxing over $\mathrm{CaH}_{2}$ under dry argon atmosphere and subsequent distillation prior to use. ${ }^{1} \mathrm{H}$ NMR spectra were recorded with a Bruker DPX-400 spectrometer (Bruker, Switzerland). Deuterated chloroform (99.8\%) and deuterated methanol (99.8\%) were used as solvents. Spectra were analyzed with MestReNova 7.0.3 software (Mestrelab Research SL, Spain) and calibrated using solvent signals $\left(\mathrm{CDCl}_{3}, 7.26 \mathrm{ppm} ; \mathrm{CD}_{3} \mathrm{OD}, 3.31 \mathrm{ppm}\right)$. The molecular weights of the polymers were obtained from ${ }^{1} \mathrm{H}$ NMR calculations. Dynamic light scattering (DLS) measurements were performed on a Malvern Zetasizer Nano S (ZEN 1600) with NP samples directly from the microfluidic assembly at $25^{\circ} \mathrm{C}$. The distribution curve was calculated with a CONTIN fit for random distribution. Cumulant analysis was performed on the correlation function, and the mean intensity was collected for static light scattering interpretation. Transmission electron micrographs were acquired through an FEI CM 200 microscope operating at an acceleration voltage of $200 \mathrm{kV}$. Polymeric NP samples were dissolved to $1 \mathrm{wt} \%$ in distilled water and dropped onto a copper grid covered with a nitroglycerin film coated with carbon. A staining agent ( $2 \%$ uranyl acetate) was added.

\subsection{Syntheses of copolymers R-PMOXA-PDMS-PMOXA-R and hydrophobized phthalocyanine zinc complex (H-PcZn) 7}

\subsubsection{Syntheses of copolymers R-PMOXA-PDMS-PMOXA-R}

The copolymers R-PMOXA-PDMS-PMOXA-R were synthesized as follows, following the main synthetic lines reported in Refs. [34,40-42].

2.2.1.1. Synthesis of copolymer Pip- $A_{7} B_{73} A_{7}$-Pip. To a solution of Poly(dimethylsiloxane), bis(hydroxyalkyl) terminated (5.6 g, $1 \mathrm{mmol})$, in toluene $(25 \mathrm{~mL})$ was added 2,6 -lutidine $(0.35 \mathrm{~mL}, 3 \mathrm{mmol})$, followed by addition of a solution of trifluoromethanesulfonic anhydride $(0.4 \mathrm{~mL}, 2.4 \mathrm{mmol})$ in hexane $(2.5 \mathrm{~mL})$, drop wise in an ice bath under argon. The reaction mixture was stirred at constant temperature for $3 \mathrm{~h}$. Then the solvent was removed under vacuum to give an active 
intermediate as yellowish oil. The oil was dissolved in a mixed solvent of chloroform (15 mL) and acetonitrile (20 mL). Monomer 2-methyl-oxazoline ( $1.3 \mathrm{~mL}, 15 \mathrm{mmol}$ ) was added at room temperature. The reaction mixture was heated to $40^{\circ} \mathrm{C}$ and stirred at constant temperature for $24 \mathrm{~h}$. Then the reaction was quenched with solid piperazine $(1.29 \mathrm{~g}, 15 \mathrm{mmol})$ at r.t. Solvent was removed by evaporation to yield a residue. The residue was purified by ultra-filtration with cellulose regenerated membrane (Millipore, cut off at $5 \mathrm{kDa}$ ) using ethanol/water $(80: 20, \mathrm{v} / \mathrm{v}$, at least for four times. In the first time, a saturated solution of $\mathrm{NaHCO}_{3}(5 \mathrm{~mL})$ was added to remove acidic side product) as eluent to produce $6.0 \mathrm{~g}$ compound Pip- $\mathrm{A}_{7} \mathrm{~B}_{73} \mathrm{~A}_{7}-\mathrm{Pip}$ as a slightly yellowish solid.

Yield: 86\%. ${ }^{1} \mathrm{H}$ NMR $\left(400 \mathrm{MHz}, \mathrm{CDCl}_{3}\right): \delta 3.20-3.66\left(\mathrm{~m}, 64 \mathrm{H},-\mathrm{CH}_{2}-\mathrm{O}-\mathrm{CH}_{2}-+-\mathrm{CH}_{2}-\mathrm{NAc}-+-\mathrm{NAc}-\mathrm{CH}_{2}-\mathrm{CH}_{2}-\mathrm{NAc}-+\right.$ $\left.-\mathrm{NAc}-\mathrm{CH}_{2}-\right), 2.52-2.84\left(\mathrm{~m}, 2 \mathrm{H},-\mathrm{CH}_{2}-\mathrm{N}\left(-\mathrm{CH}_{2}-\mathrm{CH}_{2}-\right)_{2} \mathrm{NH}\right), 2.02-2.22\left(\mathrm{~m}, 42 \mathrm{H},-\mathrm{NC}(\mathrm{O}) \mathrm{CH}_{3}\right), 1.62\left(\mathrm{~m}, 4 \mathrm{H},-\mathrm{SiMe}_{2}-\right.$ $\left.\mathrm{CH}_{2}-\mathrm{CH}_{2}-\right), 0.58\left(\mathrm{~m}, 4 \mathrm{H},-\mathrm{SiMe}_{2}-\mathrm{CH}_{2}-\mathrm{CH}_{2}-\right),-0.04$ to $0.26\left(\mathrm{~m}, 444 \mathrm{H},-\mathrm{Si}\left(\mathrm{CH}_{3}\right)_{2}-\right) \mathrm{ppm}$.

2.2.1.2. Synthesis of copolymer $\mathrm{HOOC}-A_{7} B_{73} A_{7}-\mathrm{COOH}$. To a solution of copolymer Pip- $\mathrm{A}_{7} \mathrm{~B}_{73} \mathrm{~A}_{7}$-Pip $(0.7 \mathrm{~g})$ in dichloromethane $(5 \mathrm{~mL})$ was added succinic anhydride $(60 \mathrm{mg})$ at room temperature under argon. The reaction mixture was heated to reflux and stirred at same temperature for $24 \mathrm{~h}$. Solvent was removed by evaporation to afford a residue. The residue was purified by ultra-filtration with cellulose-regenerated membrane (Millipore, a cut off at $5 \mathrm{kDa}$ ) using ethanol/water (80:20, v/v, at least for four times) as eluent to produce compound HOOC- $\mathrm{A}_{7} \mathrm{~B}_{73} \mathrm{~A}_{7}-\mathrm{COOH}(0.68 \mathrm{~g})$ as a slightly yellowish solid.

Yield: 94\%. ${ }^{1} \mathrm{H}$ NMR (400 MHz, $\left.\mathrm{CD}_{3} \mathrm{OD}\right): \delta 3.36-3.73\left(\mathrm{~m}, 72 \mathrm{H},-\mathrm{CH}_{2}-\mathrm{O}-\mathrm{CH}_{2}-+-\mathrm{CH}_{2}-\mathrm{NAc}-+-\mathrm{NAc}-\mathrm{CH}_{2}-\mathrm{CH}_{2}-\mathrm{NAc}-+\right.$ $\left.-\mathrm{NAc}-\mathrm{CH}_{2}-,-\mathrm{N}\left(-\mathrm{CH}_{2}-\mathrm{CH}_{2}-\right)_{2} \mathrm{NC}(\mathrm{O})-\right), 2.45-2.71\left(\mathrm{~m}, 2 \mathrm{H},-\mathrm{CH}_{2}-\mathrm{N}\left(-\mathrm{CH}_{2}-\mathrm{CH}_{2}-\right)_{2} \mathrm{NC}(\mathrm{O})-\mathrm{CH}_{2}-\mathrm{CH}_{2}-\mathrm{C}(\mathrm{O}) \mathrm{OH}\right), 2.13(\mathrm{~m}$, $\left.42 \mathrm{H},-\mathrm{NC}(\mathrm{O}) \mathrm{CH}_{3}\right), 1.61\left(\mathrm{~m}, 4 \mathrm{H},-\mathrm{SiMe}_{2}-\mathrm{CH}_{2}-\mathrm{CH}_{2}-\right), 0.57\left(\mathrm{~m}, 4 \mathrm{H},-\mathrm{SiMe}_{2}-\mathrm{CH}_{2}-\mathrm{CH}_{2}-\right),-0.03$ to 0.28 (m, $444 \mathrm{H},-\mathrm{Si}$ $\left.\left(\mathrm{CH}_{3}\right)_{2}-\right)$ ppm.

\subsubsection{Synthesis of hydrophobized phthalocyanine zinc complex (H-PcZn) 7}

Water soluble phthalocyanine zinc complex 4 [43] $(80 \mathrm{mg}, 0.07 \mathrm{mmol})$ was dissolved in DMF and DCC $(88.7 \mathrm{mg}$, $0.43 \mathrm{mmol})$ was added, followed by addition of NHS $(49.2 \mathrm{mg}, 0.43 \mathrm{mmol})$. The combined organic mixture was stirred at r.t. for $24 \mathrm{~h}$. Then solid was filtered and washed with a small amount of DMF. Dry ether $(20 \mathrm{~mL})$ and dichloromethane $(5 \mathrm{~mL})$ were added to the above solution. The mixture was centrifuged for $5 \mathrm{~min}$. Solid was collected and the solid was mixed with ether $(8 \mathrm{~mL})$ and dichloromethane $(2 \mathrm{~mL})$. The mixture was centrifuged again to provide the active intermediate 5 ( $55 \mathrm{mg}$ ) which can be used for the next step with further purification. To a solution of 5 ( $50 \mathrm{mg}, 0.03 \mathrm{mmol}$ ) in DMF was added poly (dimethylsiloxane) monoaminopropyl terminated 6 ( $155 \mathrm{mg}, 0.14 \mathrm{mmol})$ and solid $\mathrm{K}_{2} \mathrm{CO}_{3}(19.4 \mathrm{mg}, 0.14 \mathrm{mmol})$. The reaction mixture was stirred at r.t. for $24 \mathrm{~h}$. Then solid was removed and washed with a small amount of DMF. The combined DMF was removed under high vacuum to give a residue. The residue was purified by flash chromatography on silica gel using dichloromethane/methanol $(\mathrm{v} / \mathrm{v}, 20 / 1)$ as an eluent to give compound $\mathbf{7}(140 \mathrm{mg})$ as a dark blue slurry.

Yield: $80 \% .{ }^{1} \mathrm{H}$ NMR $\left(400 \mathrm{MHz}, \mathrm{CDCl}_{3}\right): \delta 6.74-8.20(\mathrm{~m}, 28 \mathrm{H}, \mathrm{Ar}-\mathrm{H}), 3.50\left(\mathrm{~m}, 8 \mathrm{H},-\mathrm{CH}_{2}-\mathrm{CH}_{2}-\mathrm{NH}-\mathrm{CO}-\right), 1.45-1.75(\mathrm{~m}$, $\left.16 \mathrm{H}, \mathrm{CH}_{3}-\mathrm{CH}_{2}-\mathrm{CH}_{2}-\mathrm{CH}_{2}-,-\mathrm{CH}_{2}-\mathrm{CH}_{2}-\mathrm{CH}_{2}-\mathrm{NH}-\right), 1.25$ (m, 8H, $\left.\mathrm{CH}_{3}-\mathrm{CH}_{2}-\mathrm{CH}_{2}-\right), 0.82$ (t, $12 \mathrm{H}, 8.0 \mathrm{~Hz}, \mathrm{CH}_{3}-\mathrm{CH}_{2}-$ ), $0.48\left(\mathrm{~m}, 16 \mathrm{H},-\mathrm{CH}_{2}-\mathrm{CH}_{2}-\mathrm{Si}-,-\mathrm{Si}-\mathrm{CH}_{2}-\mathrm{CH}_{2}-\right),-0.1$ to $0.1(\mathrm{~m}, 336 \mathrm{H}) \mathrm{ppm}$.

\subsection{UV Absorbance measurement}

The wavelength-dependent absorption spectrum of the materials was measured from 680 to $900 \mathrm{~nm}$ using a spectrophotometer (DU 730, Beckman Coulter), which in turn was used as an input spectrum for spectral unmixing of PA signals.

\subsection{Multispectral optoacoustic tomography (MSOT) experiments in parameters and protocol}

Photoacoustic (PA) imaging on phantoms and mice was carried out on an inVision 256-TF (iThera Medical GmbH, Munich, Germany) MSOT system as described previously by others [44]. Pulsed near infrared (NIR) light, at a wavelength adjustable by a parametric oscillator pumped by an Nd: YAG laser provides excitation from 680 to $980 \mathrm{~nm}$ with a pulse duration of $10 \mathrm{~ns}$ and repetition rate of $10 \mathrm{~Hz}$. Light from a fiber bundle is divided into 10 output arms to uniformly illuminate the sample all around its circumference in the imaging plane. A 256-TF array of concave transducer elements covering a circular arc of $270^{\circ}$ was used in receive mode only, to detect PA signals with $5 \mathrm{MHz}$ central frequency. During image acquisition, the phantom or mouse were submerged in a water bath maintained at a constant temperature of $37^{\circ} \mathrm{C}$, and translated through the imaging plane.

Cylindrical agar (1.5\% w/w, Sigma) scattering (1\% intralipid, Sigma) phantoms ( $2 \mathrm{~cm}$ diameter) described previously [45] were molded to contain two inner cylindrical channels $(3 \mathrm{~mm}$ in diameter) in which the probe and control samples were loaded. The phantoms were then translated through the imaging plane and PA imaging was performed every $2 \mathrm{~mm}$ at six different z-positions using wavelengths in the range $680-850 \mathrm{~nm}$ with $10 \mathrm{~nm}$ steps with 4 averaged frames per wavelength. These measurements were carried out on a wide range of concentrations to determine the linearity of the MSOT signal with probe concentration, as well as to understand the limit of detection of the probes. Controls consisted of classical black ink with an optical density of 0.07 at $680 \mathrm{~nm}$ to serve as an intensity measurement reference. Images were reconstructed with model-based tomographic reconstruction [46], spectrally unmixed using linear regression with two components, where one spectrum was that of a flat uniform absorber to represent ink, and the other was the absorption spectrum of the probe as measured by the spectrophotometer (Spectramax plus, Molecular Devices). 


\subsection{Animal preparation}

Animal handling was performed in compliance with protocols approved by the Bavarian Animal Research Authority. 8 -week old female CD-1 albino mice were injected intravenously via tail vein with $200 \mu$ of probe. Whole- body NIR fluorescence imaging was performed on clipped animals under anesthesia ( $2 \%$ isofluorane). Imaging was performed at 5 min and 24 h post-injection.

Animal imaging was performed under anesthesia using isoflurane in oxygen. A single cross-section of the mouse containing liver, spleen and kidneys was imaged non-stop before, during and after the probe injection to monitor probe biodistribution in these organs over time. To spectrally assess probe in vivo, 20 averages were acquired per wavelength at 680,700 , $760,770,800$ and $850 \mathrm{~nm}$. Model-based reconstruction was carried out, followed by linear regression for the probe and blood. The baseline image was subtracted in all images at all time points and a region of interest (ROI) was drawn around each organ of interest to measure over time the spectrally unmixed probe signal. Once in vivo imaging was done, the mice were sacrificed for validation purposes with a lethal dose of ketamine/xylazine and frozen to $-80{ }^{\circ} \mathrm{C}$. The thoraco-abdominal region was embedded in O.C.T (TisueTeck) and frozen. The embedded tissue was then cryosliced every $50 \mu \mathrm{m}$ in a modified Leica cryotome combined with a CCD camera to capture the fluorescent signal from the probe as described by others [47].

\section{Results and discussion}

\subsection{Syntheses of PMOXA-b-PDMS-b-PMOXA copolymer and hydrophobized phthalocyanine zinc complex (H-PcZn)}

Two amphiphilic copolymers, based on the hydrophilic polymethyloxazoline (PMOXA) block and the hydrophobic polydimethylsiloxane (PDMS) block, with the basic structure PMOXA- $b$-PDMS- $b$-PMOXA were synthesized. Both molecules have the same length ratio of the PMOXA and the PDMS blocks, but differently charged terminal groups. Fig. 1 illustrates the chemical structure of the two ABA copolymers (Pip- $A_{7} B_{73} A_{7}$-Pip and HOOC- $A_{7} B_{73} A_{7}-C O O H$ ), in which the ratio of the length of PDMS to PMOXA is 73:7, and the termini are a negatively charged carboxyl group and a positively charged amino group, respectively. The copolymers were synthesized by living cationic polymerization and a related functional transformation (Scheme 1, see Materials and methods for details).

On the other hand, the synthesis of the hydrophobized phthalocyanine zinc complex was depicted in Scheme 2. To enhance the efficacy of encapsulation of the photosensitizer as a payload for the copolymer PMOXA- $b$-PDMS- $b$-PMOXA based nanoparticle, a tetra-PMDS modified phthalocyanine zinc complex 7 was designed. For the synthesis, we started with the water soluble phthalocyanine zinc complex 4 as reported by Soper and colleagues in 2007 [43]. Briefly, compounds pentyl 4-hydroxybenzoate (1) and 4-nitrobenzene-1, 2-dinitrile (2) were used as starting materials at a basic condition to form an important intermediate 3, 4 equivalents of which reacted with Zinc salt using DBU as base under reflux to provide the compound 4. Second, N-Hydroxysuccinimid (NHS) was coupled to the carboxylic groups of $\mathbf{4}$ to produce activated OSu ester 5 that reacted with mono amino substituted PDMS $\mathbf{6}$ under a basic condition to achieve the hydrophobized phthalocyanine zinc complex 7. The PDMS tails enhance miscibility with the hydrophobic PDMS micelle core. Compound 7 is well soluble in different organic solvents, such as ethanol, dichloromethane, hexane.

\subsection{Formation of self-assemblies of amphiphilic PMOXA-PDMS-PMOXA copolymer}

As we previously reported, the hydrophobic core of the PMOXA- $b$-PDMS- $b$-PMOXA micelles is well suited for the encapsulation of hydrophobic molecules. In addition, the hydrophobized phthalocyanine zinc complex 7 should be compatible with the PMOXA- $b$-PDMS- $b$-PMOXA due to the PDMS segments of complex 7. Using the 'bulk' method for preparing nanoparticles, amphiphilic copolymer Pip- $A_{7} B_{73} A_{7}$-Pip and complex 7 (2.5 wt\%) ethanol solutions (using the minimum amount of ethanol needed for dissolution) were dropped into, and then mixed using continuous stirring in 1 XPBS buffer and formed self-assemblies. A representative TEM image is shown in Fig. 2a (micelles named P-NPs). The combination of HOOC- $\mathrm{A}_{7} \mathrm{~B}_{73} \mathrm{~A}_{7}-\mathrm{COOH}$ and complex 7 shows similar results (Fig. 2b) (micelles named N-NPs). The diameter of the resulting micelles were around $20-100 \mathrm{~nm}$ calculated from DLS measurement. The zeta potential $(\zeta)$ was a positive for P-NPs $(+29.0 \mathrm{mV})$ but negative $(-43.8 \mathrm{mV})$ in N-NPs. The result is consistent with the charge modification of the terminal groups of the triblock copolymer PMOXA- $b$-PDMS- $b$-PMOXA. Surface zeta potential is well known to have an impact on binding to plasma proteins, cells, and may influence the in vivo bio-distribution of the resulting nanoparticles. Previous studies of the cellular uptake trials have proved that zinc complex is efficiently encapsulated in the core of the copolymer PMOXA- $b$ PDMS- $b$-PMOXA based micelles with little impact on interaction with cells [34]. Therefore, the resulting micelles solution can be used directly for in vivo application without further process steps.

\subsection{UV-vis absorbance, and PA signals of the resulting nanoparticles}

In order to obtain PAI signals from the resulting nanoparticles P-NPs and N-NPs, optical absorption properties were analyzed which are known to strongly correlate with the PA signal. As shown in Fig. 3, both nanoparticles at a concentration of $30 \mathrm{mg} / \mathrm{mL}$ have the same absorption peak at $680 \mathrm{~nm}$, with an optical density (OD) 8.9 and 7.4 for P-NPs and N-NPs, 

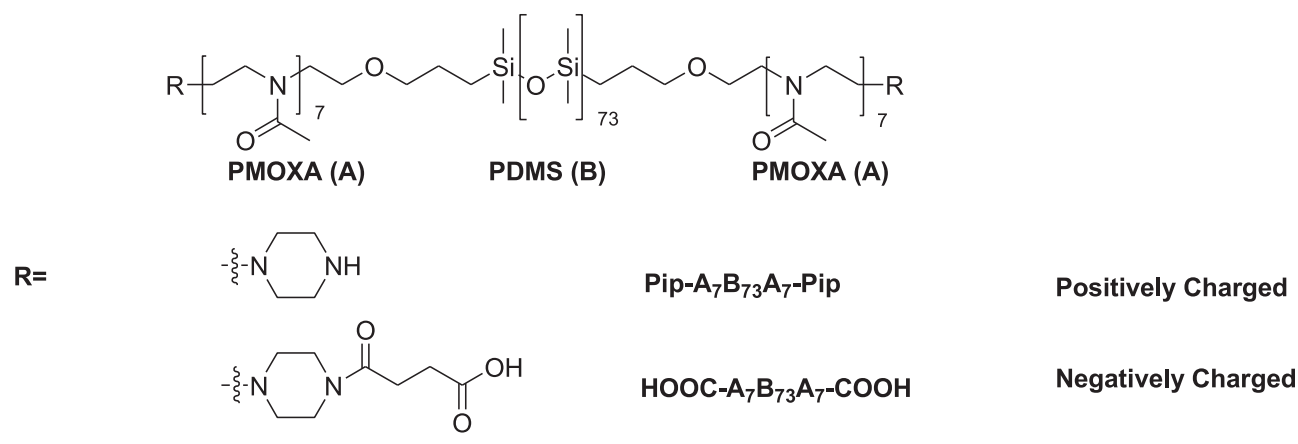

Fig. 1. Chemical structure of triblock copolymers Pip- $A_{7} B_{73} A_{7}-\mathrm{Pip}$ and HOOC- $A_{7} B_{73} A_{7}-C O O H$.

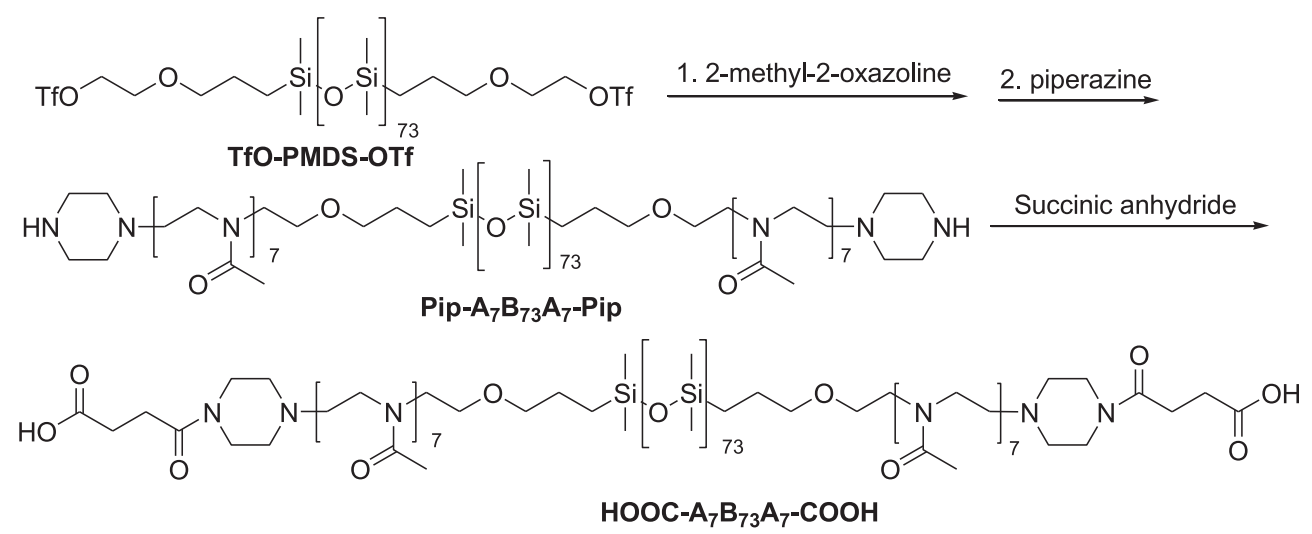

Scheme 1. Syntheses of triblock copolymers Pip- $A_{7} B_{73} A_{7}-$ Pip and HOOC- $A_{7} B_{73} A_{7}-C O O H$.

respectively. In addition, the two contrast agents exhibit a general decreasing trend in absorbance with increasing wavelength in the near infrared (NIR) range from 680 to $900 \mathrm{~nm}$, which are normally chosen for PAI owing to lower tissue absorption in this region.

PA activity of the resulting nanoparticles was then assessed using phantom measurements in a range of wavelength from 680 to $850 \mathrm{~nm}$. As depicted in Fig. 4, a $2 \mathrm{~cm}$ cylindrical agar phantom contains 2 cylindrical insertions with a diameter of $3 \mathrm{~mm}$, in which a contrast agent and a control agent, an ink at a concentration that was close to background noise, can be placed to measure the PA signal. After sequential processes including $\mathrm{x}$-section multi-spectrally scanning, image reconstruction and multispectral unmixing, an individual contribution of the contrast agent can be plotted as a function of concentration. Therefore, an optimized relationship (linear correlation) between PA intensity and the concentration of each contrast agents based on least-squares regression can be provided. As reported by previous studies that similar trends in waveform are typically seen in absorbance and PA intensity, a laser pulsing at the peak around $680 \mathrm{~nm}$ was selected for the detection of the micelles P-NPs and N-NPs [8,9]. In Fig. 5, we find that the intensities of the signals of PAs for both micelles P-NPs and N-NPs increase along with the increase of the concentration of nanoparticles (Fig. 5A and C). In certain concentration range, the intensity of MSOT signal is linear with the logarithm of concentration of the nanoparticle with $\mathrm{R}^{2}=0.997$ for P-NPs and $\mathrm{R}^{2}=0.975$ for N-NPs, respectively (Fig. 5B and D). These observations are consistent with previous reports [8,9]. Meanwhile, the lower limit of quantification (LLOQ) of P-NPs in phantoms was $0.125 \mathrm{OD} / 0.83 \mathrm{mg} / \mathrm{ml}$ in the imaging plane at depth. Comparing with P-NPs, the N-NPs have a better LLOQ $(0.06$ OD/0.53 mg/ml).

In our previous work [34], toxicity studies related to PMOXA- $b$-PDMS- $b$-PMOXA based micelles exhibited good biocompatibility in vitro and in vivo. In order to accelerate the clinical application of the nano-material and for enhanced understanding of elimination and degradation mechanisms of such nanoparticles with different terminal groups in a structure-function relationship, it is important to visualize the whole-body biodistribution of nanoparticles and simultaneously to quantify them as far as possible by MSOT. Such information allows elucidating elimination pathways and optimal dosing strategies. Here, in terms of the feasibility of the two nanoparticles for MSOT imaging by phantom analysis, we injected solutions of P-NPs and N-NPs intravenously in CD-1 albino mice and monitored their real-time distribution in the liver, spleen, kidneys, intestines using noninvasive PA imaging. At 45 min post-injection, P-NP and N-NP PA signals were detected in the spleen, liver and kidneys, as shown in the background-subtracted images in Fig. 6A. This finding was confirmed by fluorescent images of cryosections of the same mouse after it was sacrificed (Fig. 6B). Here, the strong signals in the intestines that can also be seen are typically related to autofluorescence of food but may also partly be due to the 
<smiles>CCCCCOC(=O)c1ccc(O)cc1</smiles><smiles>N#Cc1ccc([N+](=O)[O-])cc1C#N</smiles><smiles>[R16]CCCCC</smiles><smiles>CCCCCOC(=O)c1ccc(Oc2ccc(C#N)c(C#N)c2)cc1</smiles><smiles>C/C=C/CN(C)C(C)(C)C</smiles>

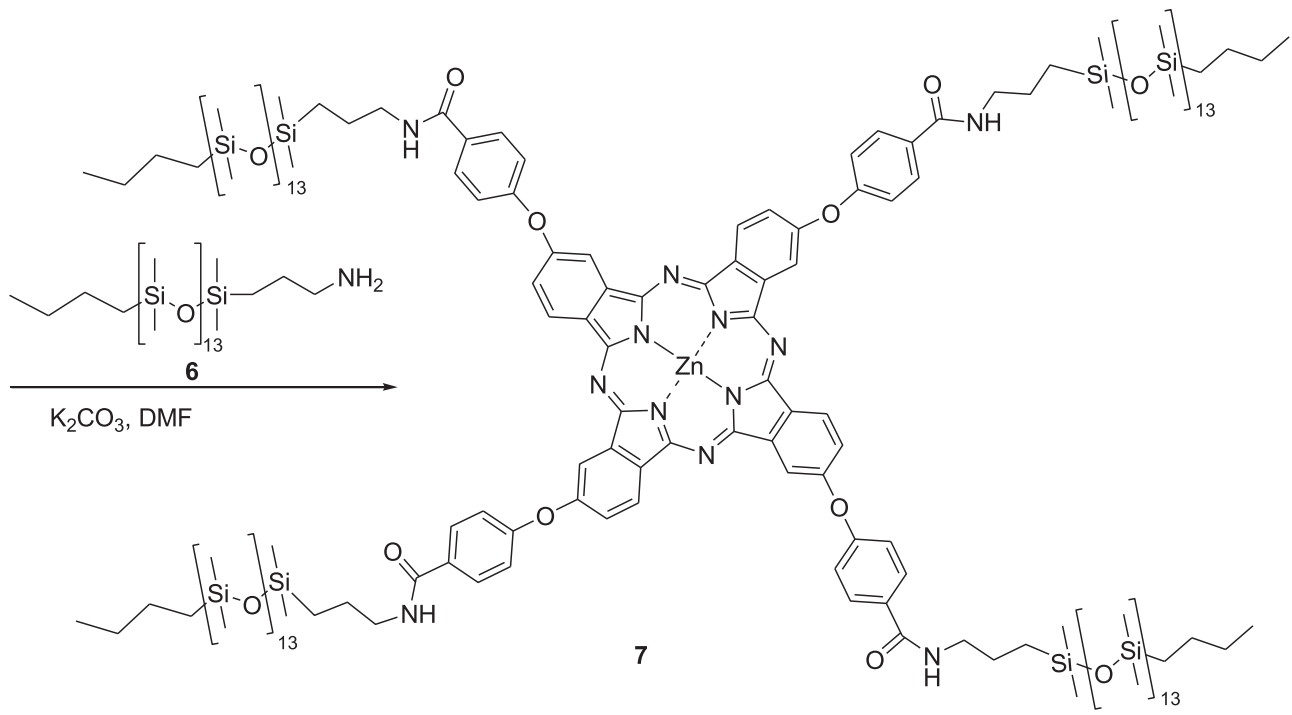

Scheme 2. Synthesis of hydrophobized phthalocyanine zinc complex 7.

excreted probe through the liver. For further quantification of the material over time, a region of interest (ROI) was drawn around each clearance organ to measure over time the spectrally unmixed signal, as shown in Fig. 7A for P-NP and Fig. 7C for N-NP. The result is illustrated in Fig. 7B for P-NP and Fig. 7D for N-NP, respectively. We found a strong accumulation of both the materials in the spleen during the 45 min of imaging with an early peak. Early liver uptake was observed mainly with P-NP followed by apparent redistribution to other organs. The amount of P-NPs in the renal cortex and renal pelvis remained at a low level as compared to that in spleen and liver (Fig. 7C).

$\mathrm{N}-\mathrm{NP}$ organ concentration reached a plateau within $10 \mathrm{~min}$, and accumulated to a significantly reduced degree in liver and kidney compared to the spleen, and compared to P-NP (Fig. 7D). Stronger nonspecific binding for positively charged materials has been reported by others [48]. These findings show a different biodistribution pattern of nanoparticledelivery compared to the free phthalocyanine zinc complex biodistribution reported by Olivo et al. [8,9] who found a dominant liver uptake. This indicates that imaging of the reticuloendothelial system may be one direct application of this system. 


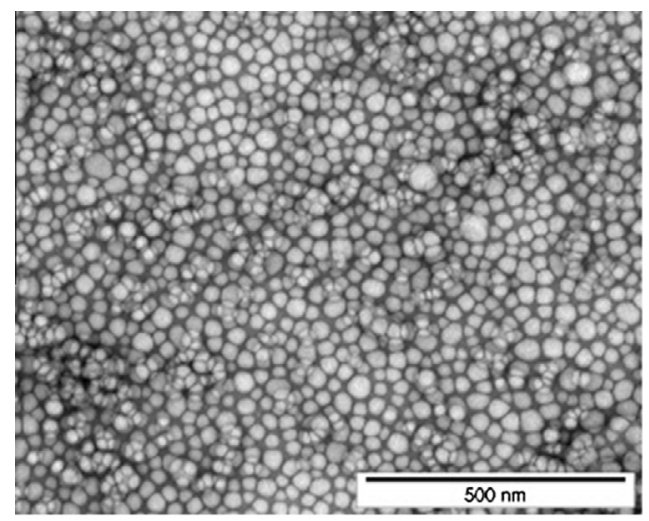

(a)

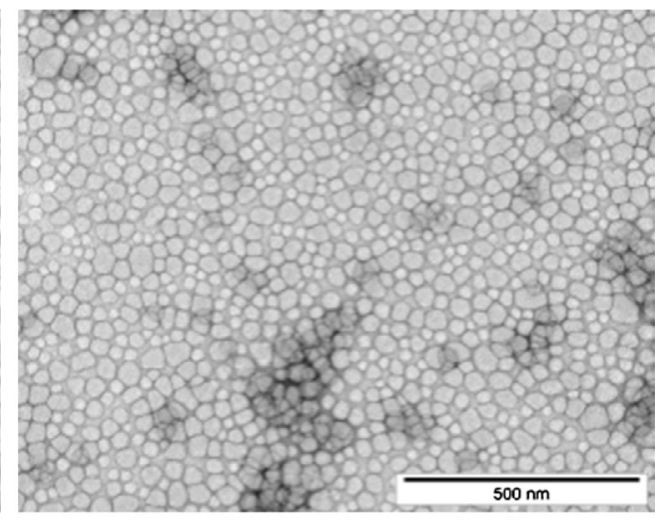

(b)

Fig. 2. TEM image of micelles of amphiphilic copolymers and the hydrophobized phthalocyanine zinc complex 7. (a) Micelles P-NPs: Pip- $A_{7} B_{73} A_{7}-P i p$ and 7; (b) Micelles N-NPs: $\mathrm{HOOC}-\mathrm{A}_{7} \mathrm{~B}_{73} \mathrm{~A}_{7}-\mathrm{COOH}$ and 7.

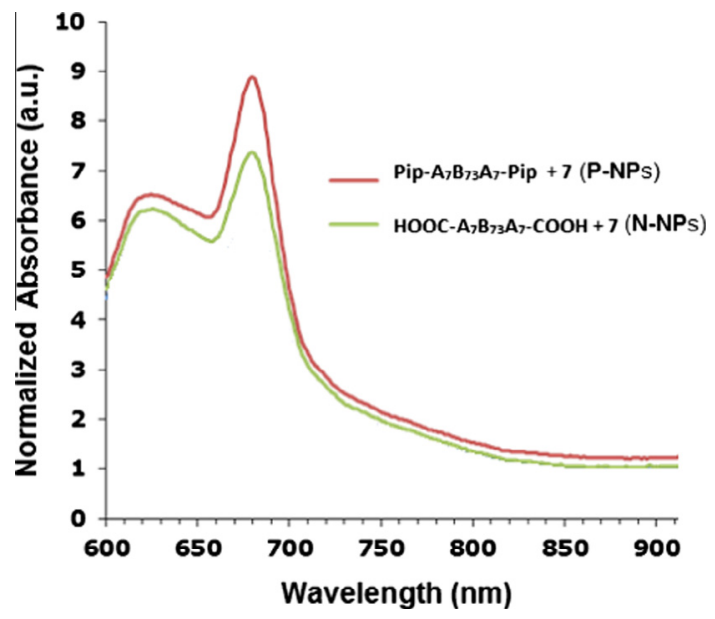

Fig. 3. UV-vis absorption spectra of micelles P-NPs and N-NPs at a concentration of $30 \mathrm{mg} / \mathrm{mL}$.

A

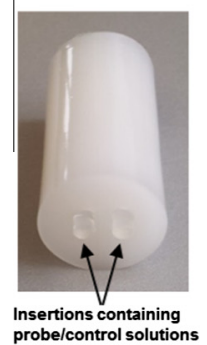

B

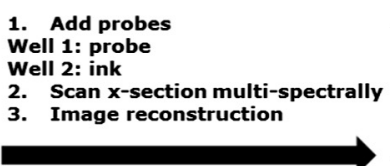

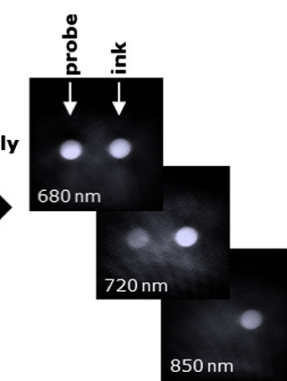

C

4. Multispectral unmixing $\begin{gathered}\text { Probe and ink } \\ \text { unmixed signal } \\ \text { concentration }\end{gathered}$ concentration

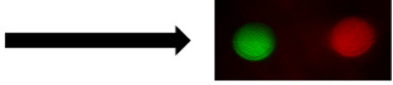

Fig. 4. Phantom imaging. A: Agar phantom with two cylindrical insertions. B: Tomographic images of a representative cross-section of the phantom with insertions. C: The reconstructed image in B is spectrally unmixed to reveal the probe and ink components.

There was no overall signal decay during the limited observation window suggesting the time frame to clear the probe might be longer than that assessed in this pilot study. From the literature [49] and our own data (manuscript in preparation) we know that such materials are eliminated by biologic pathways and are biodegradable. Thus, in summary, the strong MSOT signals from both nanoparticles P-NPs and P-NPs observed in organs suggests that MSOT imaging, combined with PDT and drugs encapsulation, offers a novel theranostic approach with high translational potential. 

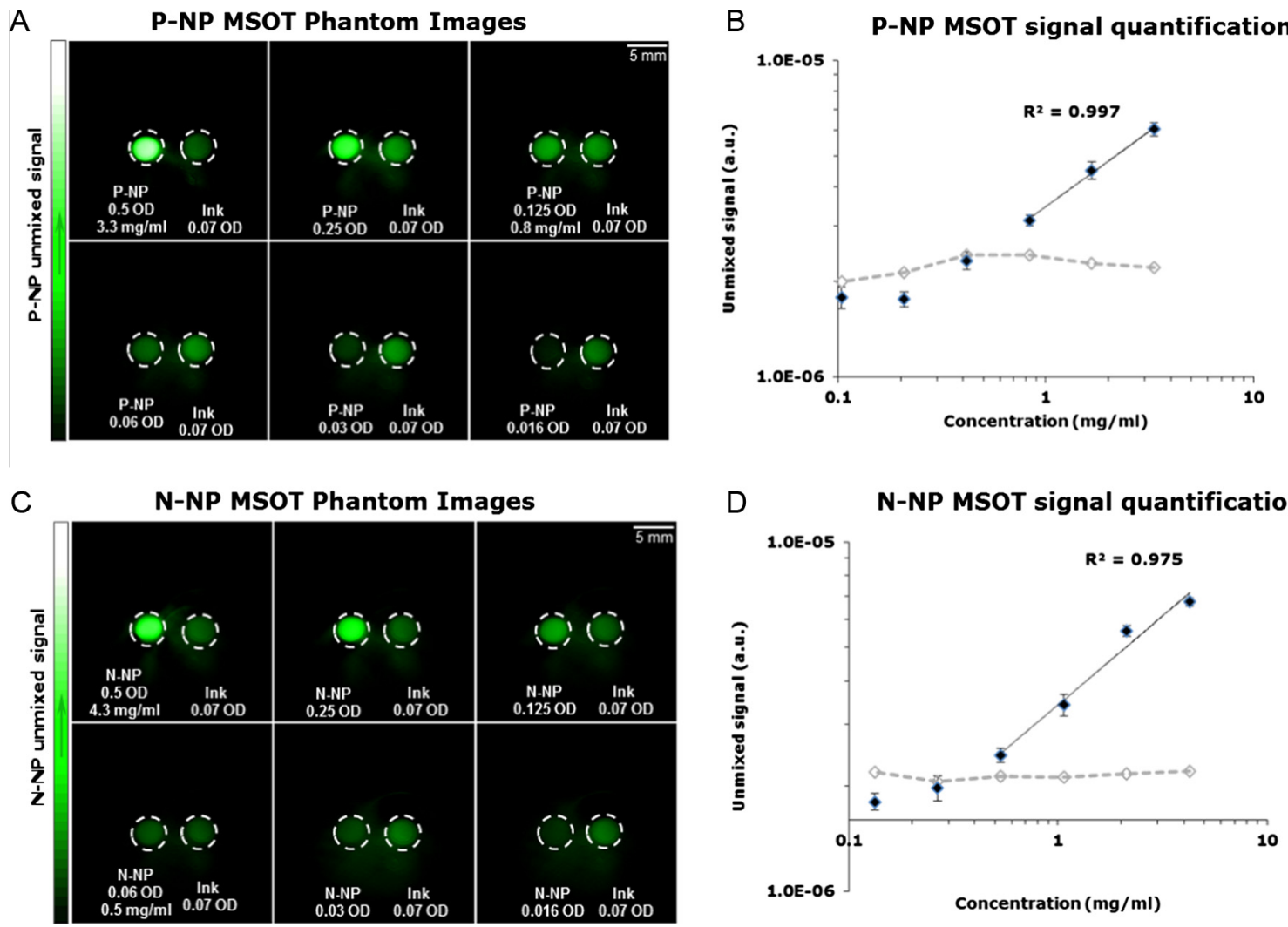

Fig. 5. MSOT phantom images of P-NPs and N-NPs and correlations between their PA signal quantifications and their concentrations. Two fold serial dilutions of P-NPs and N-NPs were inserted in a scattering agar-phantom (1.5\% agar and Intralipid; $2 \mathrm{~cm}$ diameter) along with ink $0.07 \mathrm{OD}$. MSOT imaging was performed in six imaging planes ( $~ 800 \mu \mathrm{m}$ cross-section) with a $2 \mathrm{~mm}$ step. A and C: reconstructed tomographic images depicting micelles and ink signals detected by MSOT in single cross-sections for each dilution, with laser pulsing at $680 \mathrm{~nm}$. B and D: photoacoustic signal quantification and background noise (1XPBS buffer solution, dashed grey line).
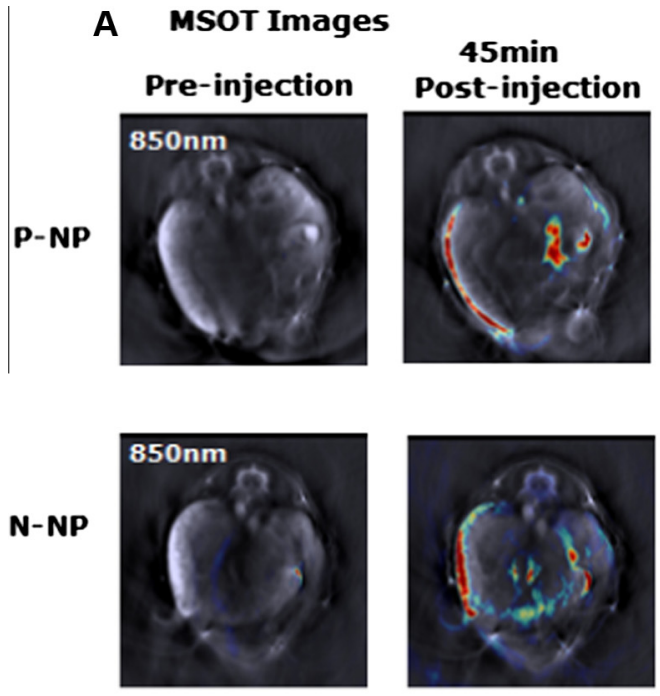

B Cryosections Fluorescence

at $1 \mathrm{~h}$
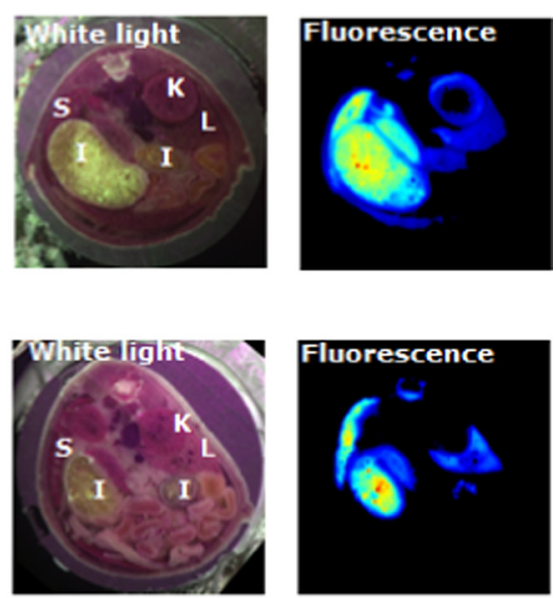

S=Spleen; I=Intestine; $K=$ Kindey; L=Liver

Fig. 6. MSOT images and the fluorescence images after cryosections. A: In vivo MSOT images from probes P-NP and N-NP at the time of pre-injection and 45 min post-injection, respectively, B: Ex vivo Fluorescence images from probes P-NP and N-NP after cryosections. 


\section{A P-NP ROIS}

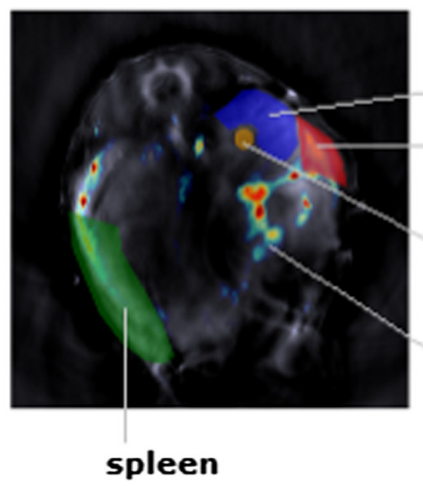

renal cortex

liver

renal pelvis

intestine

\section{N-NP ROIs}

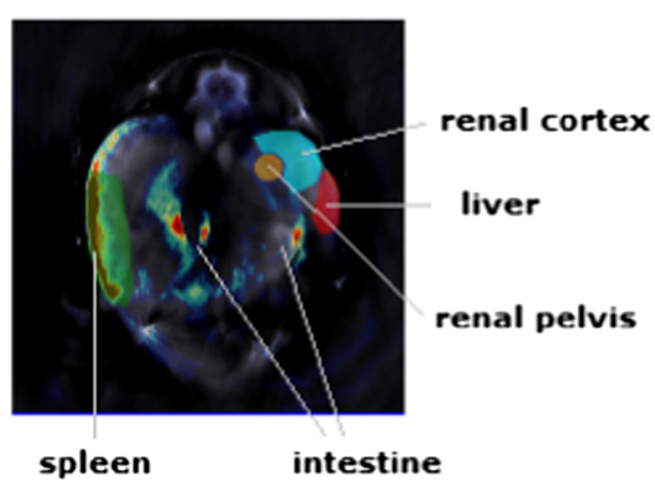

B P-NP Quantification over time

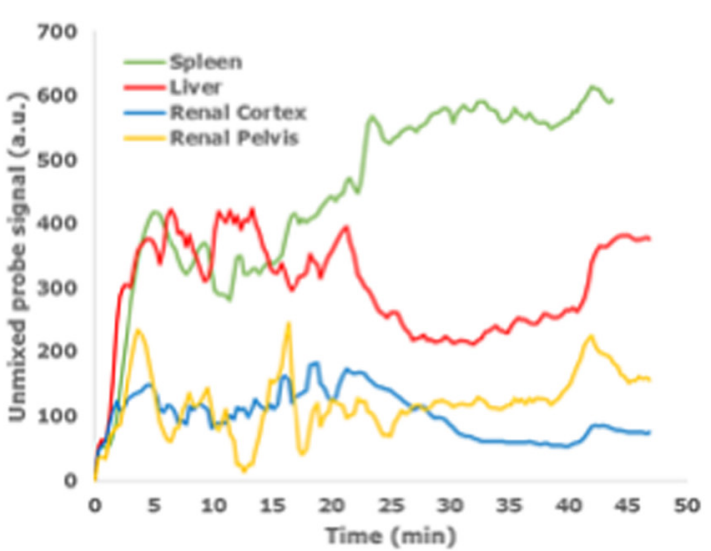

D N-NP Quantification over time

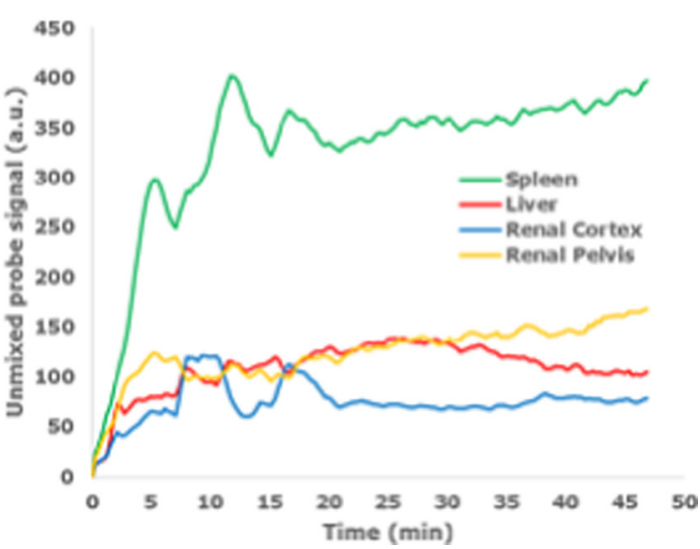

Fig. 7. ROIs and PA signal quantification over time. A and C: ROIs for P-NPs and N-NPs; B and D: PA signal quantification of nanoparticles P-NPs and N-NPs over time.

\section{Conclusion}

In summary, novel polymeric nanosystems for multispectral near-infrared photoacoustic in vivo imaging were developed, characterized and validated in vivo. Facile synthesis of two amphiphilic PMOXA- $b$-PDMS- $b$-PMOXA copolymers with different terminal functional groups through the use of living cationic polymerization and a related functional transformation was achieved, followed by self-assembly of nanoparticles in PBS buffer solution. Photoacoustic functionality was achieved by the photonic material hydrophobilized phthalocyanine zinc complex (H-PcZn), which was synthesized and efficiently encapsulated in the hydrophobic core of the two amphiphilic PMOXA- $b$-PDMS- $b$-PMOXA nanosystems. The resulting near-infrared light-responsive nanoparticles P-NPs and N-NPs exhibited powerful photoacoustic activity in phantom experiments. In vivo evaluation supported their potential as PA contrast agents: within one hour after injection, imaging and semiquantitative analysis of the biodistribution of the two nanoparticles showed tissue-specific accumulation in the spleen, liver and kidney by tomographic and non-invasive PA imaging of CD-1 albino mice. The result suggests that the main clearance route for both nanoparticles was the spleen. In addition, the positively charged nanoparticles P-NPs showed stronger tissue signals than the negatively charged N-NPs. Surface charge also had a significant impact on differential biodistribution, opening the way for developing application- or organ-specific imaging agents.

By describing the synthetic route and supramolecular self-assembly of functional polymeric nanomaterials suited for multispectral photoacoustic imaging, this study prepares the path to broader use of this interesting novel in vivo imaging technique. In future studies, the spectrum of polymer functionalities will be tailored towards even stronger differential organ binding.

In the future, polymer chemistry used in this work will be expanded by coupling different fluorescent groups covalently to the polymer backbone, allowing the study of the differential impact of covalent versus non-covalent payloads in polymeric nanosystems. Also, additional ultrasound-enhancing concepts like a light triggered fluorocarbon payload are compatible and may be further be explored with our nanomaterials [39]. 
On the application side, biodistribution, elimination and toxicity of the resulting nanoparticles will be assessed, and advanced disease models (including cancer models) will be used to establish the value of this approach in specific diseases.

\section{Acknowledgements}

The authors acknowledge Ms. V. Oliveri (Microscopy Center, University of Basel) for her kind support in taking TEM micrographs of NPs and Ms. X. Li Blatter, Dr T. Sharp (Biophsics center, university of Basel) for their kind support in DLS study as well as Dr Thomas C.P. Sardella (iThera Medical) for help with photoacoustic imaging. Financial support of Claude \& Giuliana Foundation and the Swiss National Science Foundation (NFP62 project) is gratefully acknowledged.

\section{References}

[1] V. Ntziachristos, D. Razansky, Molecular imaging by means of multispectral optoacoustic tomography (MSOT), Chem. Rev. 110 (5) (2010) $2783-2794$.

[2] L. Nie, X. Chen, Structural and functional photoacoustic molecular tomography aided by emerging contrast agents, Chem. Soc. Rev. 43 (20) (2014) $7132-7170$.

[3] J. Yao, L.V. Wang, Photoacoustic microscopy, Laser Photonics Rev. 7 (5) (2013) 758-778.

[4] S. Zackrisson, S.M. van de Ven, S.S. Gambhir, Light in and sound out: emerging translational strategies for photoacoustic imaging, Cancer Res. 74 (4) (2014) 979-1004.

[5] L.V. Wang, S. Hu, Photoacoustic tomography: in vivo imaging from organelles to organs, Science 335 (6075) (2012) 1458-1462.

[6] B.J. Vakoc, D. Fukumura, R.K. Jain, B.E. Bouma, Cancer imaging by optical coherence tomography: preclinical progress and clinical potential, Nat. Rev. Cancer 12 (5) (2012) 363-368.

[7] I. Stoffels, S. Morscher, I. Helfrich, U. Hillen, J. Lehy, N.C. Burton, et al, Metastatic status of sentinel lymph nodes in melanoma determined noninvasively with multispectral optoacoustic imaging, Sci. Transl. Med. 7 (317) (2015) 317ra199.

[8] A.B. Attia, G. Balasundaram, W. Driessen, V. Ntziachristos, M. Olivo, Phthalocyanine photosensitizers as contrast agents for in vivo photoacoustic tumor imaging, Biomed. Opt. Express 6 (2) (2015) 591-598.

[9] C.J. Ho, G. Balasundaram, W. Driessen, R. McLaren, C.L. Wong, U.S. Dinish, et al, Multifunctional photosensitizer-based contrast agents for photoacoustic imaging, Sci. Rep. 4 (2014) 5342.

[10] J.G. Fujimoto, C. Pitris, S.A. Boppart, M.E. Brezinski, Optical coherence tomography: an emerging technology for biomedical imaging and optical biopsy, Neoplasia (New York, NY) 2 (1-2) (2000) 9-25.

[11] G. Ku, L.V. Wang, Deeply penetrating photoacoustic tomography in biological tissues enhanced with an optical contrast agent, Opt. Lett. 30 (5) (2005) 507-509.

[12] R.O. Esenaliev, A.A. Karabutov, A.A. Oraevsky, Sensitivity of laser opto-acoustic imaging in detection of small deeply embedded tumors, IEEE J. Sel. Top. Quantum Electron. 5 (4) (1999) 981-988.

[13] K. Miki, T. Inoue, Y. Kobayashi, K. Nakano, H. Matsuoka, F. Yamauchi, et al, Near-infrared dye-conjugated amphiphilic hyaluronic acid derivatives as a dual contrast agent for in vivo optical and photoacoustic tumor imaging, Biomacromolecules 16 (1) (2015) 219-227.

[14] R.A. Kruger, R.B. Lam, D.R. Reinecke, S.P. Del Rio, R.P. Doyle, Photoacoustic angiography of the breast, Med. Phys. 37 (11) (2010) 6096-6100.

[15] N.C. Burton, M. Patel, S. Morscher, W.H. Driessen, J. Claussen, N. Beziere, et al, Multispectral opto-acoustic tomography (MSOT) of the brain and glioblastoma characterization, Neuroimage 65 (2013) 522-528.

[16] A. Buehler, M. Kacprowicz, A. Taruttis, V. Ntziachristos, Real-time handheld multispectral optoacoustic imaging, Opt. Lett. 38 (9) (2013) 1404-1406.

[17] P. Huang, J. Lin, W. Li, P. Rong, Z. Wang, S. Wang, et al, Biodegradable gold nanovesicles with an ultrastrong plasmonic coupling effect for photoacoustic imaging and photothermal therapy, Angew. Chem. Int. Ed. Engl. 52 (52) (2013) 13958-13964.

[18] Y. Liu, J. He, K. Yang, C. Yi, Y. Liu, L. Nie, et al, Folding Up of Gold Nanoparticle Strings into Plasmonic Vesicles for Enhanced Photoacoustic Imaging, Angew. Chem. Int. Ed. Engl. 54 (54) (2015) 15809-15812.

[19] L. Jing, X. Liang, Z. Deng, S. Feng, X. Li, M. Huang, et al, Prussian blue coated gold nanoparticles for simultaneous photoacoustic/CT bimodal imaging and photothermal ablation of cancer, Biomaterials 35 (22) (2014) 5814-5821.

[20] N. Lozano, W.T. Al-Jamal, A. Taruttis, N. Beziere, N.C. Burton, J. Van den Bossche, et al, Liposome-gold nanorod hybrids for high-resolution visualization deep in tissues, J. Am. Chem. Soc. 134 (32) (2012) 13256-13258.

[21] A. de la Zerda, S. Bodapati, R. Teed, S.Y. May, S.M. Tabakman, Z. Liu, et al, Family of enhanced photoacoustic imaging agents for high-sensitivity and multiplexing studies in living mice, ACS Nano 6 (6) (2012) 4694-4701.

[22] H. Gong, R. Peng, Z. Liu, Carbon nanotubes for biomedical imaging: the recent advances, Adv. Drug Deliv. Rev. 65 (15) (2013) $1951-1963$.

[23] K. Pu, A.J. Shuhendler, J.V. Jokerst, J. Mei, S.S. Gambhir, Z. Bao, et al, Semiconducting polymer nanoparticles as photoacoustic molecular imaging probes in living mice, Nat. Nanotechnol. 9 (3) (2014) 233-239.

[24] J. Song, X. Yang, O. Jacobson, L. Lin, P. Huang, G. Niu, et al, Sequential drug release and enhanced photothermal and photoacoustic effect of hybrid reduced graphene oxide-loaded ultrasmall gold nanorod vesicles for cancer therapy, ACS Nano 9 (9) (2015) 9199-9209.

[25] K.K. Ng, M. Shakiba, E. Huynh, R.A. Weersink, A. Roxin, B.C. Wilson, et al, Stimuli-responsive photoacoustic nanoswitch for in vivo sensing applications, ACS Nano 8 (8) (2014) 8363-8373.

[26] Q. Chen, X. Liu, J. Chen, J. Zeng, Z. Cheng, Z. Liu, A self-assembled albumin-based nanoprobe for in vivo ratiometric photoacoustic pH imaging, Adv. Mater. 27 (27) (2015) 6820-6827.

[27] X. Song, R. Zhang, C. Liang, Q. Chen, H. Gong, Z. Liu, Nano-assemblies of J-aggregates based on a NIR dye as a multifunctional drug carrier for combination cancer therapy, Biomaterials 57 (2015) 84-92.

[28] A. Taruttis, S. Morscher, N.C. Burton, D. Razansky, V. Ntziachristos, Fast multispectral optoacoustic tomography (MSOT) for dynamic imaging of pharmacokinetics and biodistribution in multiple organs, PLoS ONE 7 (1) (2012) e30491.

[29] D. Peer, J.M. Karp, S. Hong, O.C. Farokhzad, R. Margalit, R. Langer, Nanocarriers as an emerging platform for cancer therapy, Nat. Nanotechnol. 2 (12) (2007) 751-760.

[30] M.E. Davis, Z.G. Chen, D.M. Shin, Nanoparticle therapeutics: an emerging treatment modality for cancer, Nat. Rev. Drug Discovery 7 (9) (2008) $771-$ 782.

[31] D. Hudson, A. Margaritis, Biopolymer nanoparticle production for controlled release of biopharmaceuticals, Crit. Rev. Biotechnol. 34 (2) (2014) 161179.

[32] R. Lehner, X. Wang, S. Marsch, P. Hunziker, Intelligent nanomaterials for medicine: carrier platforms and targeting strategies in the context of clinical application, Nanomedicine 9 (6) (2013) 742-757.

[33] D. Pan, X. Cai, B. Kim, A.J. Stacy, L.V. Wang, G.M. Lanza, Rapid synthesis of near infrared polymeric micelles for real-time sentinel lymph node imaging, Adv. Healthc. Mater. 1 (5) (2012) 582-589.

[34] K. Liu, Z. Zhu, X. Wang, D. Goncalves, B. Zhang, A. Hierlemann, et al, Microfluidics-based single-step preparation of injection-ready polymeric nanosystems for medical imaging and drug delivery, Nanoscale 7 (40) (2015) 16983-16993.

[35] J. Muller, A. Wunder, K. Licha, Optical imaging, Recent Results Cancer Res. 187 (2013) 221-246.

[36] J.F. Lovell, T.W. Liu, J. Chen, G. Zheng, Activatable photosensitizers for imaging and therapy, Chem. Rev. 110 (5) (2010) $2839-2857$. 
[37] C. Lee, J. Kim, Y. Zhang, M. Jeon, C. Liu, L. Song, et al, Dual-color photoacoustic lymph node imaging using nanoformulated naphthalocyanines, Biomaterials 73 (2015) 142-148.

[38] C.A. Strassert, G.M. Bilmes, J. Awruch, L.E. Dicelio, Comparative photophysical investigation of oxygen and sulfur as covalent linkers on octaalkylamino substituted zinc(II) phthalocyanines, Photochem. Photobiol. Sci. 7 (6) (2008) 738-747.

[39] R.J. Paproski, A. Forbrich, E. Huynh, J. Chen, J.D. Lewis, G. Zheng, et al, Porphyrin nanodroplets: sub-micrometer ultrasound and photoacoustic contrast imaging agents, Small 12 (3) (2016) 371-380.

[40] P. Broz, S.M. Benito, C. Saw, P. Burger, H. Heider, M. Pfisterer, et al, Cell targeting by a generic receptor-targeted polymer nanocontainer platform, J. Control. Release 102 (2) (2005) 475-488.

[41] N. Ben-Haim, P. Broz, S. Marsch, W. Meier, P. Hunziker, Cell-specific integration of artificial organelles based on functionalized polymer vesicles, Nano Lett. 8 (5) (2008) 1368-1373.

[42] A. Ranquin, W. Versees, W. Meier, J. Steyaert, P. Van Gelder, Therapeutic nanoreactors: combining chemistry and biology in a novel triblock copolymer drug delivery system, Nano Lett. 5 (11) (2005) 2220-2224.

[43] V.T. Verdree, S. Pakhomov, G. Su, M.W. Allen, A.C. Countryman, R.P. Hammer, et al, Water soluble metallo-phthalocyanines: the role of the functional groups on the spectral and photophysical properties, J. Fluoresc. 17 (5) (2007) 547-563.

[44] A. Dima, N.C. Burton, V. Ntziachristos, Multispectral optoacoustic tomography at 64, 128, and 256 channels, J. Biomed. Opt. 19 (3) (2014) 36021.

[45] I. Stoffels, D. Schadendorf, S. Morscher, U. Hillen, I. Helfrich, J. Leyh, et al, Metastatic status of sentinel lymph nodes in melanoma determined noninvasively with multispectral optoacoustic imaging, Sci. Transl. Med. 7 (2015).

[46] A. Buehler, A. Rosenthal, T. Jetzfellner, A. Dima, D. Razansky, V. Ntziachristos, Model-based optoacoustic inversions with incomplete projection data, Med. Phys. 38 (3) (2011) 1694-1704.

[47] A. Sarantopoulos, G. Themelis, V. Ntziachristos, Imaging the bio-distribution of fluorescent probes using multispectral epi-illumination cryoslicing imaging, Mol. Imaging Biol. 13 (5) (2011) 874-885.

[48] E. Blanco, H. Shen, M. Ferrari, Principles of nanoparticle design for overcoming biological barriers to drug delivery, Nat. Biotechnol. 33 (9) (2015) 941951.

[49] M.E. Fox, F.C. Szoka, J.M. Frechet, Soluble polymer carriers for the treatment of cancer: the importance of molecular architecture, Acc. Chem. Res. 42 (8) (2009) 1141-1151. 